\title{
Enhanced immunotherapeutic profile of oncolytic virus-based cancer vaccination using cyclophosphamide preconditioning
}

\author{
Jonathan G Pol (1) , ${ }^{1,2,3,4,5,6,7}$ Matthew J Atherton, ${ }^{1}$ Kyle B Stephenson, ${ }^{1}$ \\ Byram W Bridle, ${ }^{8}$ Samuel T Workenhe, ${ }^{1}$ Natasha Kazdhan, ${ }^{1}$ AJ Robert McGray, ${ }^{1}$ \\ Yonghong Wan, ${ }^{1}$ Guido Kroemer, ${ }^{2,3,4,5,6,7,9,10,11}$ Brian D Lichty ${ }^{1}$
}

To cite: Pol JG, Atherton MJ, Stephenson KB, et al. Enhanced immunotherapeutic profile of oncolytic virusbased cancer vaccination using cyclophosphamide preconditioning. Journal for ImmunoTherapy of Cancer 2020;8:e000981. doi:10.1136/ jitc-2020-000981

- Additional material is published online only. To view please visit the journal online (http://dx.doi.org/10.1136/jitc2020-000981).

Accepted 22 July 2020

Check for updates

(C) Author(s) (or their employer(s)) 2020. Re-use permitted under CC BY-NC. No commercial re-use. See rights and permissions. Published by BMJ.

For numbered affiliations see end of article.

\section{Correspondence to} Dr Jonathan G Pol; pol_jonathan@yahoo.fr

Dr Brian D Lichty; lichtyb@mcmaster.ca

\section{ABSTRACT}

Despite a sizeable body of research, the efficacy of therapeutic cancer vaccines remains limited when applied as sole agents. By using a prime:boost approach involving two viral cancer vaccines, we were able to generate large tumor-specific $\mathrm{CD}^{+} \mathrm{T}$-cell responses in a murine model of disseminated pulmonary melanoma. Significant increases in the number and quality of circulating effector T-cells were documented when low-dose cyclophosphamide (CTX) was administered pre-vaccination to tumor-bearing but not tumor-free hosts. Interestingly, tumor-bearing mice receiving CTX and co-primed with a melanoma differentiation antigen together with an irrelevant control antigen exhibited significantly enhanced immunity against the tumor, but not the control antigen, in secondary lymphoid organs. This result highlighted an increased cancer-specific reactivity of vaccine-induced T-cell responses following CTX preconditioning. Additionally, an acute reduction of the frequency of peripheral regulatory T-cells (Tregs) was noticeable, particularly in the proliferating, presumably tumour-reactive, subset. Enhanced infiltration of lungs with multifunctional T-cells resulted in overt reduction in metastatic burden in mice pretreated with CTX. Despite doubling the median survival in comparison to untreated controls, most vaccinated mice ultimately succumbed to cancer progression. However, preconditioning of the virus-based vaccination with CTX resulted in a remarkable improvement of the therapeutic activity leading to complete remission in the majority of the animals. Collectively, these data reveal how CTX can potentiate specific cellular immunity in an antigenrestricted manner that is only observed in vaccinated tumor-bearing hosts while depleting replicating Tregs. A single low dose of CTX enhances antitumor immunity and the efficacy of this potent prime:boost platform by modulating the kinetics of the vaccine-specific responses. Clinical assessment of CTX combined with next-generation cancer vaccines is indicated.

\section{INTRODUCTION}

Induction of endogenous antitumor immune responses using vaccination has been investigated for decades and although progress has been gradual the full potential of this approach is yet to be realized. ${ }^{1}$ Using vaccines as sole therapeutic agents exerts limited clinical activity and major barriers to the success of vaccines include the inability of vaccines to overcome immunosuppressive cell populations, including regulatory T-cells (Tregs) and/or to mount robust responses against the targeted antigen $(\mathrm{s}) \cdot{ }^{23}$ Combining cancer vaccines with other immunomodulants is being actively pursued by many investigators with the hope that such polypharmacy will help overcome these major obstacles. ${ }^{4}$

We have developed a heterologous prime:boost cancer vaccination. ${ }^{5-7}$ The strategy relies on (1) an immune prime mediated by a replication-incompetent adenoviral vaccine administered intramuscularly, followed by (2) a boost using an oncolytic replication-competent rhabdoviral vaccine infused systemically. Both viral vectors harbor the same transgene that encodes a tumorassociated antigen (TAA). We have shown that this approach generates tumor-specific $\mathrm{CD}^{+} \mathrm{T}$-cell responses of particularly remarkable magnitudes owing to the ability of the boosting vector to directly engage splenic central memory T-cells $(\mathrm{Tcm}) .{ }^{8}$ In murine studies, such adaptive anticancer immunity results in prolonged survival and efficacy in various solid tumor models thus offering an ideal platform to study the kinetics of vaccineinduced antitumor immunity. ${ }^{5-7}$

Combining chemotherapeutic compounds with immunotherapy could be considered counterintuitive as numerous cytotoxic drugs demonstrate immunosuppressive functions. ${ }^{9}$ However, some agents have validated immunomodulatory functions particularly when administered at a low dose. Among these chemotherapeutics, cyclophosphamide (CTX) demonstrates pleiotropic effects that 
supports the induction of antitumor immunity. Briefly, CTX has been reported to (1) directly kill cancer cells in an immunogenic fashion, (2) influence dendritic cell (DC) homeostasis and promote tumor infiltration of DCs secreting more interleukin (IL)-12 and less IL-10, (3) polarize $\mathrm{CD} 4^{+} \mathrm{T}$ cells into type- 1 and/or type- 17 immune helpers, (4) stimulate the activation and proliferation of tumor-specific T lymphocytes and their infiltration inside the tumor bed and (5) reprogram and/or deplete immunosuppressive $\mathrm{T}$ regulatory cells (Tregs). ${ }^{10-12}$ However, the effect that CTX has on the dynamics of TAA-specific effector and Treg cells induced by anti-cancer vaccines has not been comprehensively characterized.

We hypothesized that combining our oncolytic vaccination approach with a single low dose of CTX would potentiate tumor-specific effector immune responses and deplete Tregs thereby favoring vaccine-mediated tumor control. This report shows that CTX preconditioning enhances the magnitude and quality of $\mathrm{CD}_{4}^{+}$and $\mathrm{CD} 8^{+}$ T-cell responses induced by prime:boost oncolytic vaccination specifically in tumor-bearing hosts, alongside focusing their reactivity toward tumor-associated rather than tumor-irrelevant antigens. CTX preconditioning leads to increased infiltration of effector T-cells within the tumor microenvironment (TME) and reduced peripheral Tregs, particularly when proliferating. Remarkably, the combination therapy translated into complete and durable disease clearance in the majority of mice treated.

\section{MATERIALS \& METHODS Mice}

Female C57BL/6 mice (8-10 weeks old at study initiation) were purchased from Charles River Laboratory (Wilmington, Massachusetts, USA) and housed in a specific pathogen-free facility.

\section{Recombinant viruses}

Ad-empty, Ad-OVA and Ad-DCT are replication-deficient adenoviruses (E1/E3-deletion) based on the human serotype 5. VSV-DCT is a replication-competent oncolytic viral agent which derives from the wild-type Indiana strain of the vesicular stomatitis virus (VSV). Ad-empty has no transgene. Ad-DCT and VSV-DCT encode the human dopachrome tautomerase (DCT). The OVA transgene encodes the chicken ovalbumin and was used as a tumorunrelated antigen control.

\section{Cell culture}

Murine melanoma B16-F10 cells (expressing the murine DCT antigen) were grown in F11-MEM containing $10 \%$ fetal bovine serum (FBS), $2 \mathrm{mM}$ L-glutamine, $1 \mathrm{mM}$ sodium pyruvate and vitamin solution, $0.01 \mathrm{mM}$ nonessential amino acids, $50 \mathrm{mM} \beta$-mercaptoethanol, $100 \mathrm{U} /$ $\mathrm{mL}$ penicillin, and $100 \mathrm{mg} / \mathrm{mL}$ streptomycin (all from Invitrogen, Grand Island, New York, USA).

\section{In vivo treatments}

For adenovirus injection, mice were anesthetized in a sealed chamber containing $5 \%$ inhalation isoflurane.
Adenoviral vectors were administered intramuscularly at a total dose of $2 \times 10^{8} \mathrm{pfu}\left(1 \times 10^{8}\right.$ plaque forming units (pfu) in $50 \mu \mathrm{L}$ phosphate-buffered saline (PBS) per thigh). VSV-DCT was injected intravenously in $200 \mu \mathrm{L}$ PBS at a dose of $2 \times 10^{9}$ pfu. CTX was administered through intraperitoneal (i.p.) injection at $50 \mathrm{mg} / \mathrm{kg}$ in $200 \mu \mathrm{L}$ of saline solution 1 day prior to Ad prime (other groups received $200 \mu \mathrm{L}$ of saline solution as control).

\section{Tumor challenge}

For lung tumor engraftment, $2.5 \times 10^{5}$ B16-F10 melanoma cells were injected intravenously in $200 \mu \mathrm{L}$ saline water. Mice were monitored daily and euthanized for tissue harvest or on signs of morbidity.

\section{Tissue homogenates}

Blood sampling was performed through retro-orbital sinus bleeding using a heparinized capillary tube. Blood was collected into a heparinized saline solution (30 U/ $\mathrm{mL})$. Peripheral white blood cells were cleared of erythrocytes in ammonium chloride potassium (ACK) buffer at room temperature for $5 \mathrm{~min}$ then washed in Hanks medium; the procedure was repeated twice. After harvest, lymph nodes and spleen were crushed between glass slides to release leukocytes. Splenic red blood cells were lysed in ACK buffer as described for blood. Lungs bearing melanoma metastases were dissociated enzymatically using a cocktail of $0.05 \%$ collagenase, $0.002 \%$ hyaluronidase and $0.02 \%$ DNAse I. Lung-infiltrating leukocytes were isolated using the EasySep Mouse CD90.2 Positive Selection Kit II (Stemcell Technologies, Vancouver, British Columbia, Canada) following manufacturer's recommendations.

\section{Peptides}

Peptides corresponding to the immunodominant epitopes of DCT: (1) DCT ${ }_{180-188}$ SVYDFFVWL/"SVY" that binds to $\mathrm{H}-2 \mathrm{~K}^{\mathrm{b}} ; 100 \%$ conserved between human and murine DCT and (2) DCT ${ }_{89-102}$ KFFHRTCKCTGNFA/"KFF" from human DCT that binds to I-A ${ }^{\mathrm{b}}$; differing from the murine epitope in position 92: His (human) $\rightarrow$ Asn (murine). Peptides corresponding to the immunodominant epitopes of OVA: (1) OVA $_{257-264}$ SIINFEKL/"SIIN" that

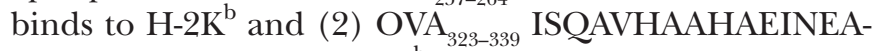
GR/ "ISQ" that binds to I-A $A^{\mathrm{b}}$. Peptides were synthesized by Biomer Technologies (San Francisco, California, USA).

\section{Antibodies}

Monoclonal antibodies used in flow cytometry assays: anti-CD16/CD32 (clone 2.4G2) to block Fc receptors, anti-CD3 (clone 145-2 C11), anti-CD8 $\alpha$ (clone 53-6.7), anti-CD4 (clone RM4-5), anti-CD25 (clone PC61) and anti-FoxP3 (clone FJK-16s) for detecting cell surface and intranuclear markers, and anti-interferon $\gamma($ IFN $\gamma)$ (clone XMG1.2) and anti-tumor necrosis factor $\alpha$ (TNF $\alpha$; clone MP6-XT22) for intracellular cytokine staining. All reagents were purchased from BD Pharmingen (Mississauga, Ontario, Canada) with the exception of anti-FoxP3 that was purchased from eBioscience/Thermo Fisher Scientific (Burlington, Ontario, Canada). 


\section{Antigen-specific T-cell responses}

DCT-specific T-cell responses were measured 9 and 14 days post prime and 5 days post boost. Peripheral blood mononuclear cells (PBMCs) or splenocytes were incubated in complete Roswell Park Memorial Institute (RPMI) medium (ie supplemented with FBS $10 \%$, penicillin-streptomycin $1 \%$ and L-glutamine $1 \%$ ) with the SVY peptide $(2 \mu \mathrm{g} / \mathrm{mL})$ or KFF peptide $(15 \mu \mathrm{g} / \mathrm{mL})$ for DCTspecific $\mathrm{CD}^{+}$or $\mathrm{CD}^{+}{ }^{+} \mathrm{T}-\mathrm{cell}$ (re-)stimulation, respectively, or with the SIIN peptide $(2 \mu \mathrm{g} / \mathrm{mL})$ for OVA-specific $\mathrm{CD}^{+}$T-cell (re-)stimulation. Incubation was performed in an incubator $\left(37^{\circ} \mathrm{C}, 5 \% \mathrm{CO}_{2}, 95 \%\right.$ humidity) for 5 hours with brefeldin A $(1 \mu \mathrm{g} / \mathrm{mL}$, GolgiPlug BD Pharmingen) during the last 4 hours. Leukocytes were labeled with LIVE/DEAD fixable near-IR dye (Life Technologies/Thermo Fisher Scientific) following supplier's recommendations to identify live lymphocytes. Cells were incubated with antibodies targeting CD16/CD32 before staining with fluorescent-labeled antibodies targeting T-cell markers. Then, cells were permeabilized and fixed with Cytofix/Cytoperm (BD Pharmingen) and stained for intracellular cytokines. Data were acquired using a FACSCanto flow cytometer with FACSDiva software (BD Pharmingen) and analyzed with FlowJo v10 for Mac (Tree Star, Oregon, USA).

\section{In vivo BrdU labeling}

5-Bromo-2'-deoxyuridine (BrdU) was first administered i.p. at $50 \mathrm{mg} / \mathrm{kg}$ immediately after tumor challenge (ie, 4 days prior to CTX and 5 days prior to Ad) and maintained over the time until tissue harvest in the drinking water at $0.8 \mathrm{mg} / \mathrm{mL}$. BrdU-containing water was refreshed every day. Tregs that incorporated BrdU into their DNA during the $S$ phase of the cell cycle were stained using the APC BrdU Flow Kit (BD Pharmagen) following supplier's recommendations.

\section{Statistical analyses}

GraphPad Prism was used for graphing and statistical analyses. Immune response data were plotted used mean \pm SEM or SD. Unpaired Student's two-tailed t-test was used to compare two groups, ordinary one-way analysis of variance was used for multiple groups. Survival curves were plotted using Kaplan-Meier method, and compared using the log-rank test. Differences considered significant when $\mathrm{p} \leq 0.05(* \mathrm{p}<0.05, * * \mathrm{p}<0.01, * * * \mathrm{p}<0.001$, $* * * * \mathrm{p}<0.0001)$.

\section{RESULTS}

\section{CTX increases the magnitude and quality of circulating antigen-specific T-cells in tumor-bearing mice}

Mice bearing disseminated B16-F10 melanoma lung tumors were treated with varying combinations of vaccines encoding DCT, a well-characterized melanoma-associated antigen, with or without CTX preconditioning (figure 1A). Priming with Ad-DCT induced specific anti-DCT CD8 ${ }^{+}$ T-cells and the magnitude of the circulating responses was significantly increased by a single low dose of CTX prior to priming (figure 1B, online supplementary figure $\mathrm{S} 1$ ). Boosting with systemic oncolytic VSV-DCT increased the magnitude of $\mathrm{CD}^{+}$T-cell responses compared with Ad alone and a further significant increase in both specific $\mathrm{CD} 8^{+} \mathrm{Tc} 1$ and $\mathrm{CD} 4^{+} \mathrm{Th} 1$ cell frequencies were seen when CTX was combined with this potent prime:boost platform (figure 1C, D, online supplementary figure S1) in a tumor-specific manner as no such effect was observed in tumor-free mice (figure 1E). CTX preconditioning also increased specific IFN $\gamma$ production by both $\mathrm{CD}^{+}$and $\mathrm{CD}^{+}$T-cells when quantified using mean fluorescence intensities (figure 1F, G). Collectively, these data show that low-dose CTX improves the immunotherapeutic profile of this vaccine in a tumor-restricted manner.

\section{CTX preconditioning focuses vaccine-induced immunity against the tumor}

In order to determine the effect of CTX pretreatment with relation to tumor-antigen specificity, secondary lymphoid organs were harvested from tumor-bearing mice 9 days after co-priming with Ad-DCT plus an Ad vector expressing the tumor-unrelated xenoantigen ovalbumin (Ad-OVA). DCT-targeting and OVA-targeting immune responses were quantified using intracellular cytokine staining and flow cytometry. Within the tumordraining lymph nodes, CTX pretreatment failed to significantly alter the magnitude of OVA-specific single IFN $\gamma^{+}$ and dual $\mathrm{IFN}^{+} \mathrm{TNF}^{+} \mathrm{CD} 8^{+}$T-cell responses whereas a significant expansion was noted in both DCT-specific populations (figure 2A-D). These findings were mirrored by OVA-specific and DCT-specific $\mathrm{CD}^{+}$splenocytes (figure 2E-H). No significant effect of CTX preconditioning on the magnitude of OVA-specific $\mathrm{CD} 4^{+}$Th1-cell frequencies was observed (figure 2I). However, CTX significantly increased the corresponding DCT-specific $\mathrm{CD}^{+}$T-cell population (figure 2J). Taken together, lowdose CTX significantly improves vaccine-induced immunity against a well-defined melanocytic TAA expressed in B16-F10 cells but has no effect on the immune response against a xenoantigen that is irrelevant to the tumor.

\section{Pre-vaccination CTX increases the $\mathrm{CD8}^{+} \mathrm{T}$-cells/Tregs ratio}

As Tregs have been shown to constrain the expansion of $\mathrm{CD}^{+} \mathrm{T}$ cells, ${ }^{13}$ we hypothesized that the increased magnitude of vaccine-induced TAA-specific T-cell responses observed after low-dose CTX would be accompanied by decreased Treg proliferation. We interrogated the dynamics of various short-term circulating T-cell subsets in tumor-bearing mice following Ad-DCT \pm CTX. At 24 hours post-Ad, CTX preconditioning did not result in generalized lymphodepletion when $\mathrm{CD} 4^{+}$and $\mathrm{CD} 8^{+}$ T-cells were quantified (online supplementary figure S2). A relative expansion of $\mathrm{CD}^{+}$and $\mathrm{CD}^{+}{ }^{+}$-cell frequencies within the circulating lymphoid compartment was documented (figure 3A, B). Co-staining for CD4, CD25 and FoxP3 revealed a significant decrease in circulating Treg frequency in the CTX group (figure 3C) and ratios 
A

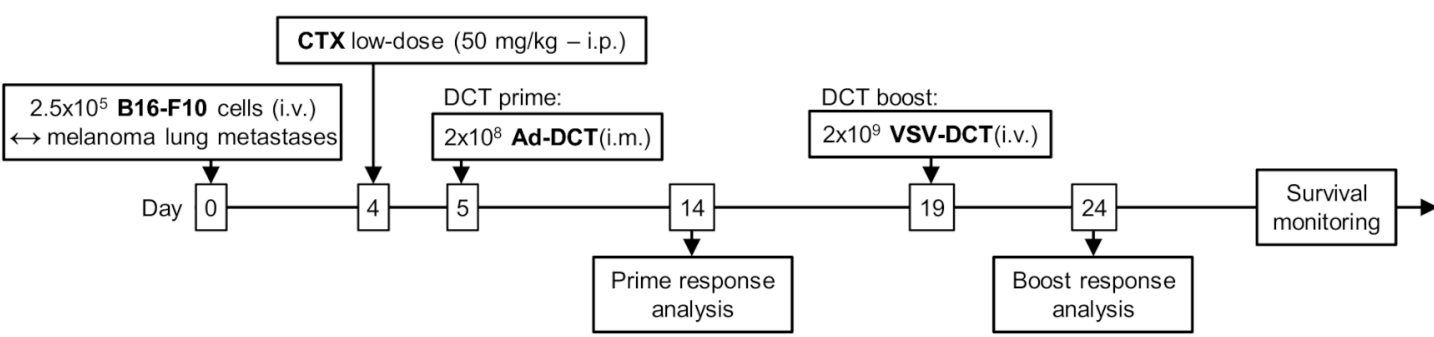

B

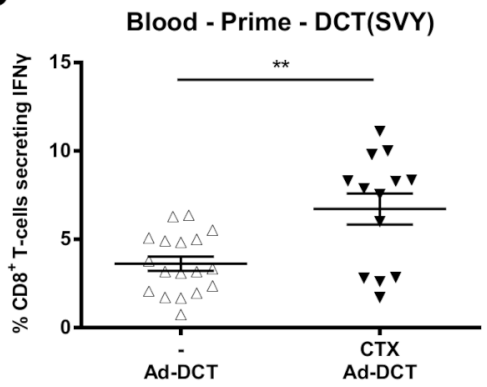

E

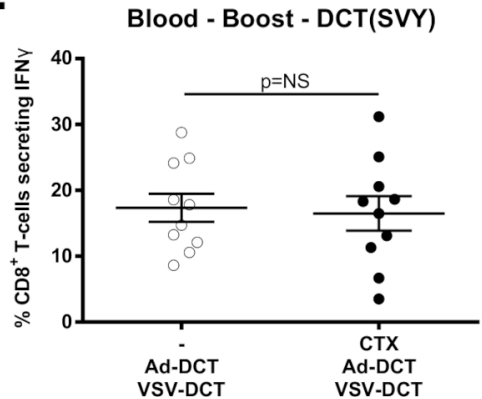

C

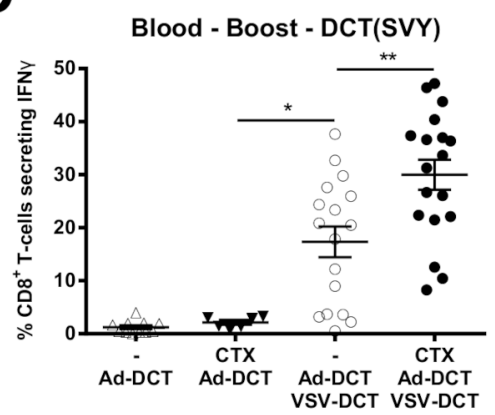

F

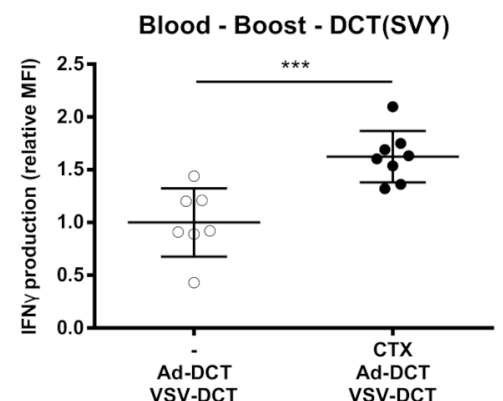

D

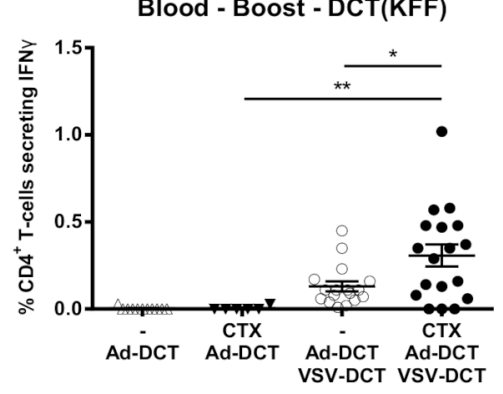

G

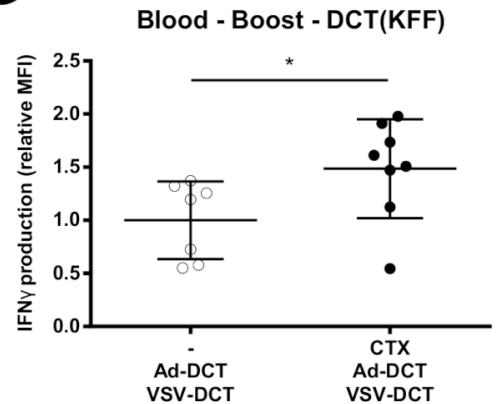

Figure 1 Cyclophosphamide (CTX) increases the magnitude and quality of virus-based vaccination in tumor-bearing mice. (A) Timeline for treatment of mice bearing B16-F10 lung tumors. To measure T-cell reactivity against the tumor-associated antigen dopachrome tautomerase (DCT), circulating lymphocytes have been restimulated ex vivo with peptides corresponding to the major histocompatibility complex (MHC)-I and MHC-II-restricted immunodominant epitopes DCT(SVY) and DCT(KFF), respectively. (B) Frequency of circulating CD8 ${ }^{+}$T-cells reacting against DCT(SVY) 9 days after priming with the adenovirus (Ad)DCT or CTX + Ad-DCT. (C) Frequency of reactive CD8 ${ }^{+}$T-cells 5 days after boosting in melanoma lung tumor-bearing animals treated with Ad-DCT, CTX + Ad-DCT, Ad-DCT + vesicular stomatitis virus (VSV)-DCT, and CTX + Ad-DCT + VSV-DCT. (D) Frequency of circulating CD4 ${ }^{+}$T-cells reacting against $\mathrm{DCT}(\mathrm{KFF}) 5$ days after boosting in tumor-bearing mice that received AdDCT, CTX + Ad-DCT, Ad-DCT + VSV-DCT, and CTX + Ad-DCT + VSV-DCT. (E) Frequency of interferon $\gamma(\text { IFN } \gamma)^{+}$CD8 ${ }^{+}$T-cells 5 days after boosting in tumor-free animals treated with Ad-DCT + VSV-DCT and CTX + Ad-DCT + VSV-DCT. (F,G) Production of IFN $\gamma$ illustrated as relative mean fluorescence intensity (MFI) in (F) DCT-specific $\mathrm{CD}_{4}^{+}$and (G) CD8 ${ }^{+}$T-cells following treatment of tumor-bearing mice with Ad-DCT + VSV-DCT and CTX + Ad-DCT + VSV-DCT. Data displayed in (B-E) consist of pools of at least two distinct experiments; dot plots indicate mean $\pm S E M$. Data displayed in $(F)$ and $(G)$ are results from one representative experiment; dot plots indicate mean \pm SD. i.m., intramuscular; i.p., intraperitoneal; i.v. intravenous.

of $\mathrm{CD}^{+}$T-cells $/ \mathrm{CD} 4^{+}$Tregs were significantly increased after preconditioning (figure 3D). BrdU was administered in the drinking water to tumor-bearing mice to quantify Treg proliferation, and blood samples were taken 72 hours post-Ad. A significant decrease in the frequency of proliferating Tregs (figure 3E) and increase in the ratio of total $\mathrm{CD} 8^{+} \mathrm{T}$ cells/BrdU ${ }^{+}$Tregs (figure $3 \mathrm{~F}$ ) in the CTX group was noted. Thus, prevaccination treatment with CTX reduced the population of proliferating Tregs resulting in a favorable proportion of effector $\mathrm{CD} 8^{+}$ $\mathrm{T}$ cells over immunosuppressive $\mathrm{CD} 4^{+}$Tregs.
Chemotherapeutic preconditioning increases lung tumor infiltration by melanoma-specific effector T-cell populations and leads to durable tumor clearance

Further analyses were performed on mice bearing lung melanoma to ascertain the functional impact of CTX on the prime:boost vaccine. In long-term survivors, marked vitiligo was observed when animals were pretreated with CTX (figure 4A). As previously reported, ${ }^{7}$ this benign autoimmune manifestation resulted from a bystander elimination of melanocytes (which express the DCT antigen) by vaccine-induced $\mathrm{CD} 8^{+}$T-cells. Lungs were 
Lung-draining lymph nodes
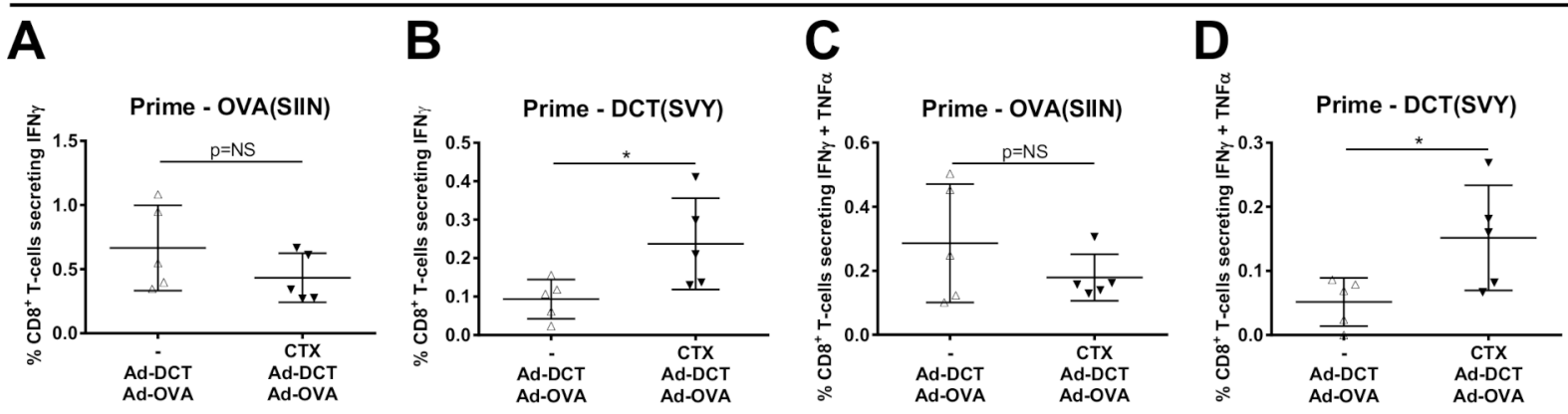

Spleen
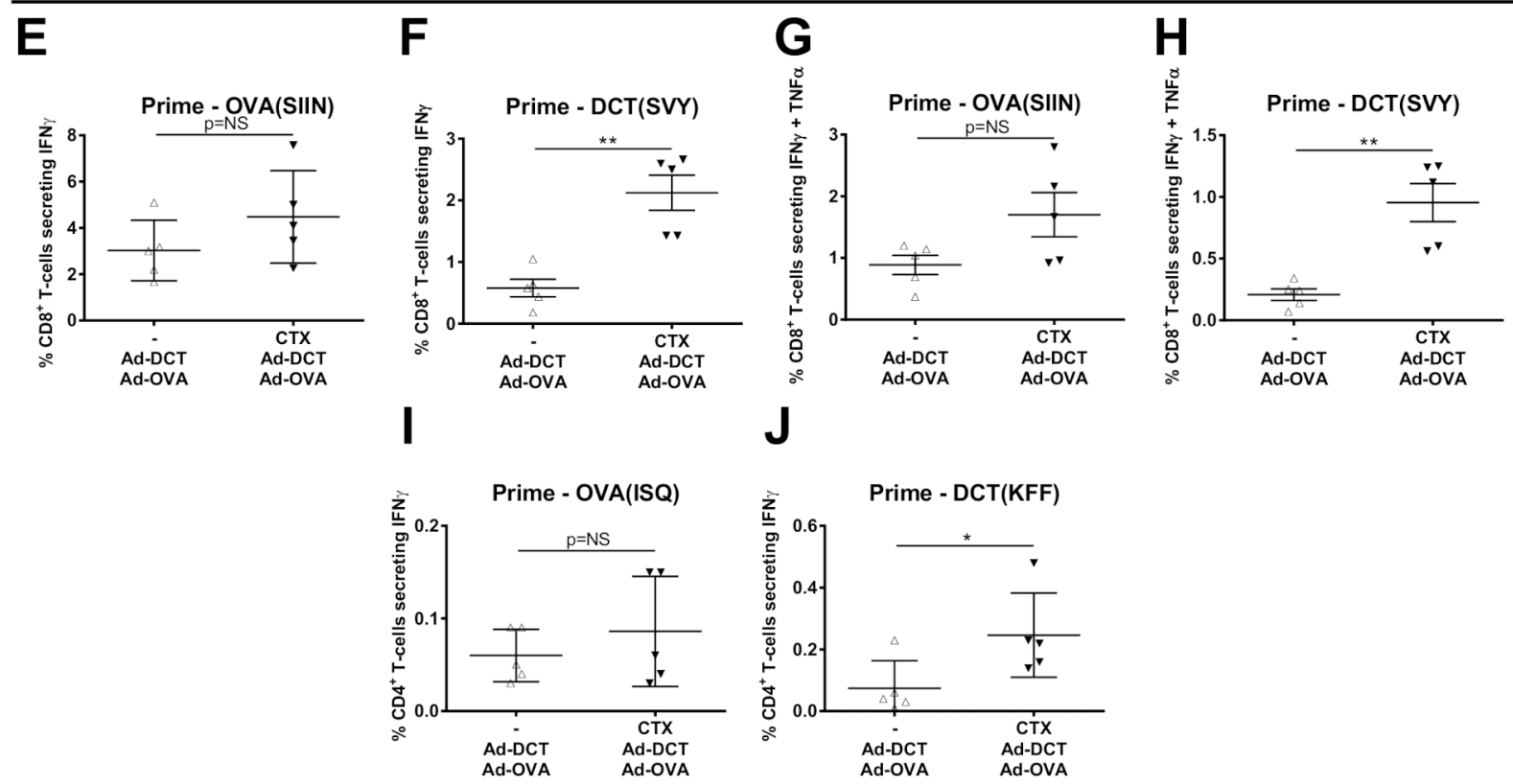

Figure 2 Preconditioning with cyclophosphamide (CTX) focuses vaccine-induced immunity against the tumor. Frequency of (A) anti-ovalbumin (OVA) interferon $\gamma(\mathrm{IFN} \gamma)^{+}$, (B) anti-dopachrome tautomerase (DCT) IFN $\gamma^{+}$, (C) anti-OVA IFN $\gamma^{+}$tumor necrosis factor $\alpha(\mathrm{TNF} \alpha)^{+}$and (D) anti-DCT IFN $\gamma^{+}$TNF $\alpha^{+} \mathrm{CD} 8^{+}$T-cells isolated from mediastinal lymph nodes draining melanoma lung metastases. Frequency of splenic (E) anti-OVA IFN $\gamma^{+},(\mathrm{F})$ anti-DCT IFN $\gamma^{+},(\mathrm{G})$ anti-OVA IFN $\gamma^{+} \mathrm{TNF} \alpha^{+}$and $(\mathrm{H})$ anti-DCT IFN $\gamma^{+}$ $\mathrm{TNF}^{+} \mathrm{CD}^{+}$T-cells. Frequency of splenic (I) anti-OVA and $(\mathrm{J})$ anti-DCT IFN $\gamma^{+} \mathrm{CD} 4^{+} \mathrm{T}$-cells. Samples harvested 9 days after covaccination with Ad-DCT and Ad-OVA with or without CTX preconditioning in mice bearing disseminated B16-F10 melanoma lung tumors. Data display mean \pm SD of one representative experiment.

harvested from tumor-bearing mice 5 days after VSV-DCT boost, alongside untreated mice, and lymphocytes were isolated. Intracellular staining of $\mathrm{CD}^{+} \mathrm{T}$-cells for IFN $\gamma$ and TNF $\alpha$ revealed significant increases of DCT-specific, single and dual positive, populations within the TME in the CTX group, whereas no reactive T-cells were detected in untreated animals (figure 4B, C). At the same time point, lungs harvested were examined for gross lesions. Pretreatment with CTX resulted in an overt reduction in tumor burden (figure 4D). Ad-DCT:VSV-DCT resulted in long-term survival in a minority of mice. However, when this regimen was preceded by a single dose of CTX, the majority of mice were cured (figure 4E). Overall, low-dose CTX increases specific antitumor immunity resulting in a remarkable survival advantage in mice bearing aggressive lung disease. In conclusion, CTX preconditioning increased infiltration of the tissues harboring melanoma lesions by polyfunctional tumor-reactive effector T-cells which preceded durable tumor control.

\section{DISCUSSION}

By preconditioning mice with a single low dose of CTX, we observed improvement of both the functionality and magnitude of effector T-cell populations in tumor-bearing mice. Intriguingly, no benefit to the immunotherapeutic profile of the vaccine was noted in tumor-free mice, nor when vaccinating melanoma-bearing mice with a tumorirrelevant antigen, uncovering an unexpected tumor/ antigen-specific function of CTX preconditioning. This report also demonstrates that CTX pretreatment impairs Treg proliferation and improves the immune profile of a potent prime:boost vaccine. Ultimately, this approach resulted in durable tumor clearance in the majority of animals bearing an aggressive pulmonary melanoma model following oncolytic prime:boost vaccination.

CTX is one of the most widely used cytotoxics within the oncology clinic. ${ }^{14}$ At high doses, CTX is used as a primary antineoplastic agent for various malignancies as well as a preconditioning agent prior to bone marrow 
A

Blood - CD4 ${ }^{+}$T-cells

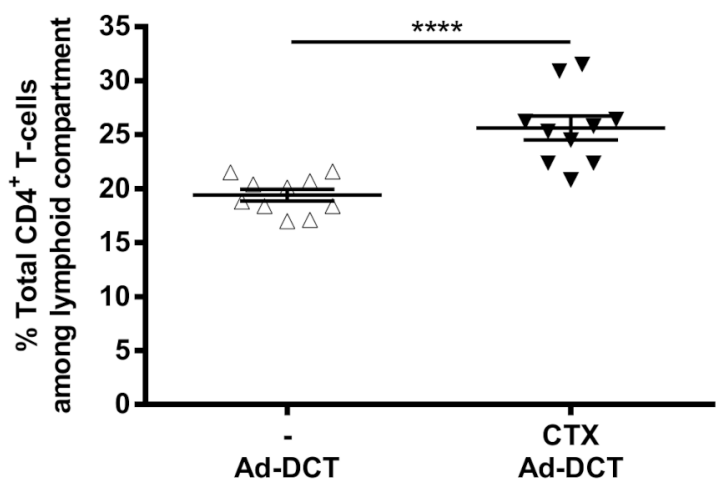

C

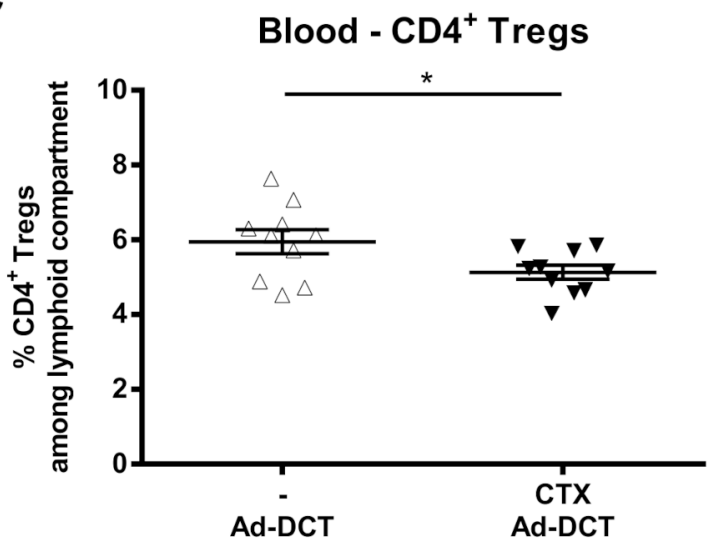

E

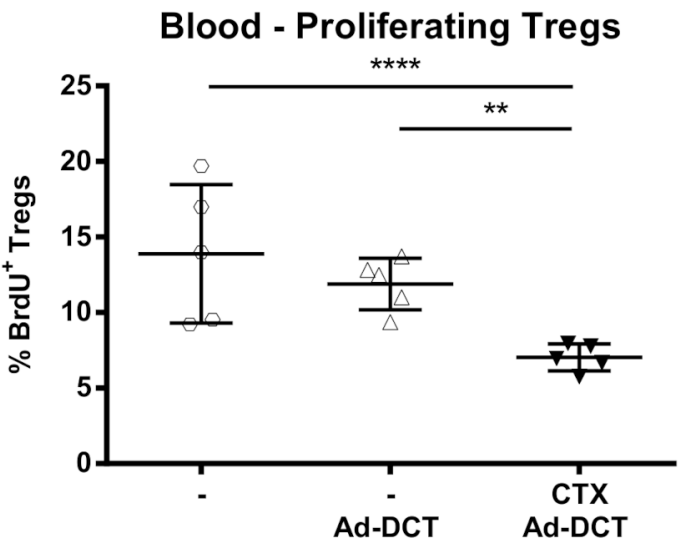

B

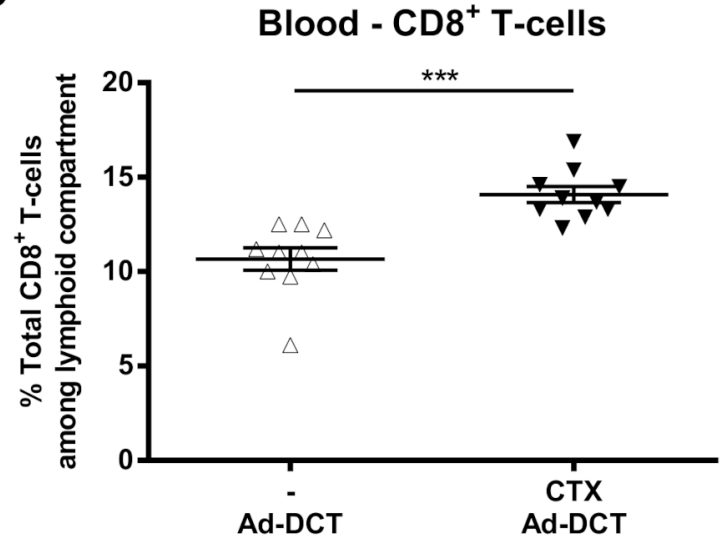

Blood - CD8 ${ }^{+}$T-cells / Tregs ratio

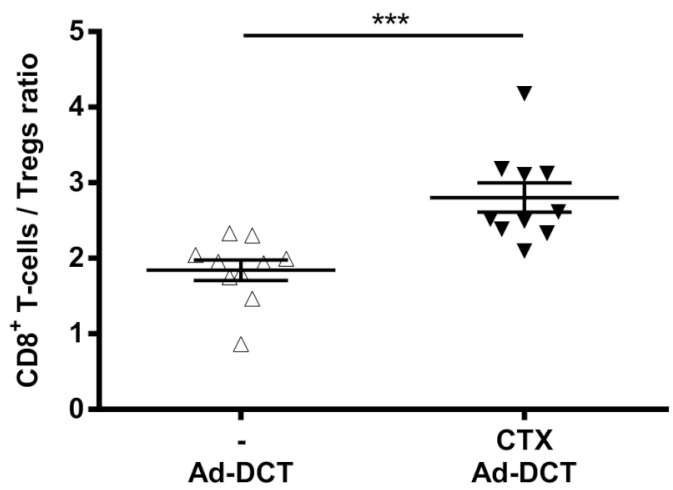

$\mathbf{F}$

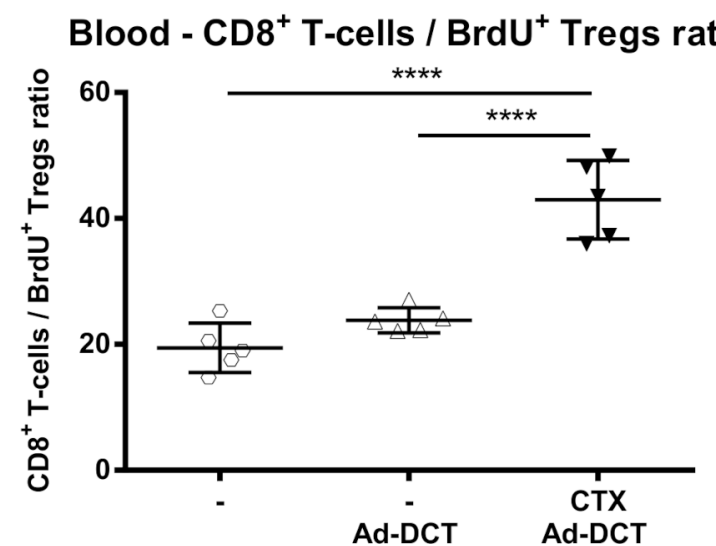

Figure 3 Pre-vaccination cyclophosphamide (CTX) increases the CD8 ${ }^{+}$T-cells/Tregs ratio and reduces proliferating Tregs in the periphery. Frequency of $(\mathrm{A})$ total CD4 ${ }^{+}$T-cells, $(\mathrm{B})$ total $\mathrm{CD} 8^{+}$T-cells and $(\mathrm{C}) \mathrm{CD}^{+} \mathrm{CD}^{+} 5^{+}$FoxP3 ${ }^{+}$Tregs among the circulating lymphoid compartment of melanoma lung-metastatic mice. (D) Ratio of CD8 ${ }^{+}$T-cells/Tregs in peripheral blood samples taken 24 hours after adenovirus (Ad)-dopachrome tautomerase (DCT), either alone or after CTX preconditioning. Graphs display mean \pm SEM of two pooled experiments. Frequency of (E) proliferating 5-bromo- $2^{\prime}$-deoxyuridine $\left(\mathrm{BrdU}^{+} \mathrm{CD}^{+} \mathrm{CD}^{+} 5^{+} \mathrm{FoxP} 3^{+}\right.$ Tregs and (F) ratio of $\mathrm{CD}^{+} \mathrm{T}$ cells/BrdU ${ }^{+}$Tregs within the lymphoid compartment of blood samples collected 72 hours after treatment of melanoma lung-metastatic animals with Ad-DCT, administered either alone or after CTX preconditioning (untreated controls labeled "-"). Mean \pm SD displayed.

transplantation achieved by ablation of CTX-sensitive populations inclusive of effector lymphocytes. ${ }^{14}$ At lower doses, CTX selectively targets $\mathrm{CD} 4^{+}$Tregs due to their low intracellular concentrations of ATP, which in turn impairs their ability to synthesize glutathione, a key molecule in the metabolic inactivation of CTX. ${ }^{15}$ This dose-dependent effect is recapitulated in this model where we document reduced Treg proliferation as well as decreased total Treg frequencies in the absence of overt circulating $\mathrm{CD} 4^{+}$and $\mathrm{CD}^{+}$lymphodepletion. 
A

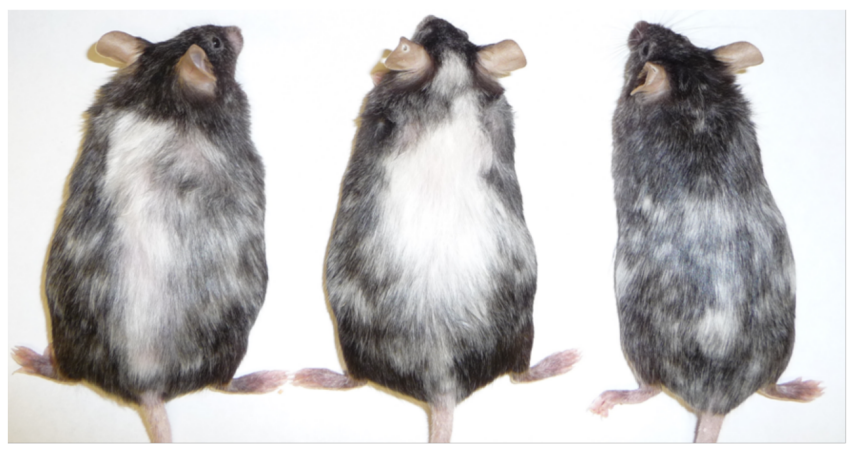

B

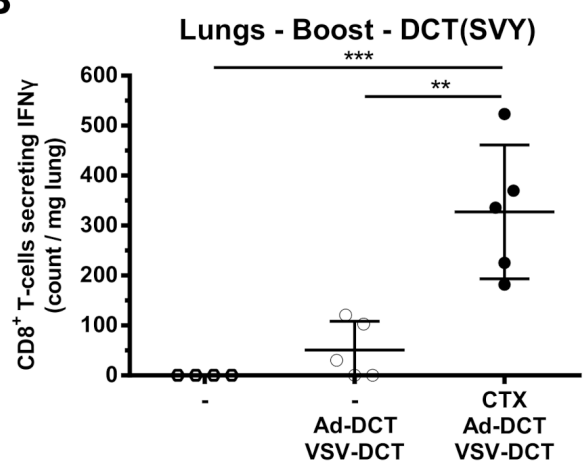

D

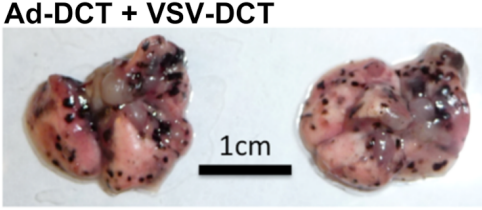

C

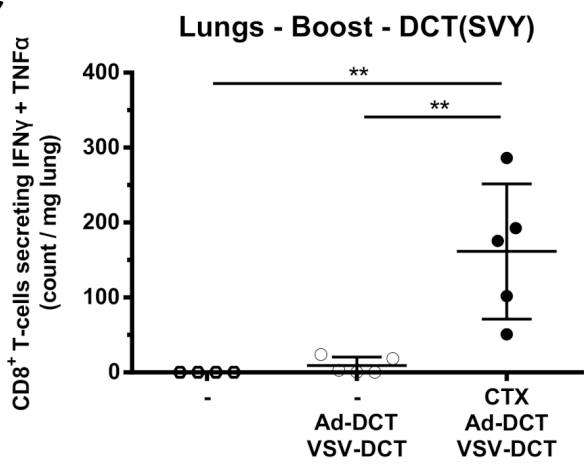

CTX + Ad-DCT + VSV-DCT
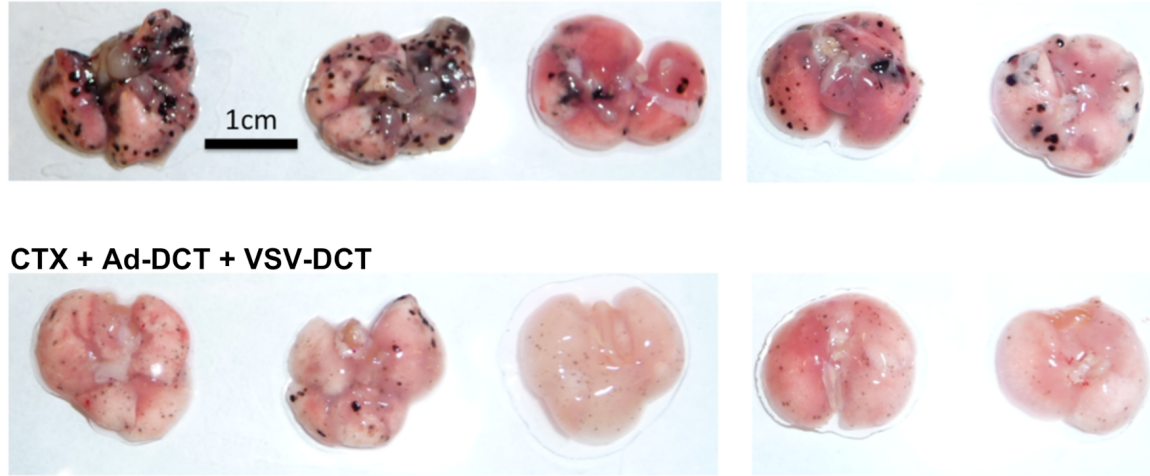

E

B16-F10 melanoma lung metastases

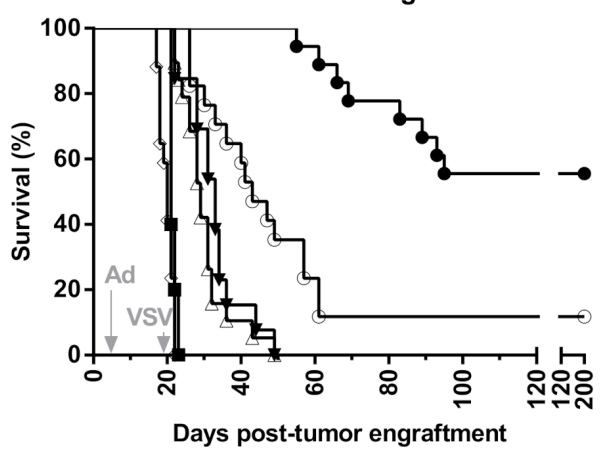

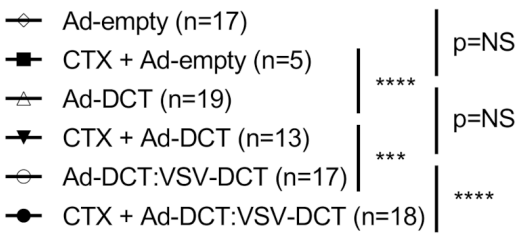

Figure 4 Chemotherapeutic preconditioning increases specific antimelanocytic effector activity. (A) Marked vitiligo observed in long-term surviving mice preconditioned with cyclophosphamide (CTX). Number of (B) interferon $\gamma(\text { IFN } \gamma)^{+}$and (C) IFN $\gamma^{+}$tumor necrosis factor $\alpha(\mathrm{TNF} \alpha)^{+}$dopachrome tautomerase (DCT)-specific $\mathrm{CD}^{+}$tumor-infiltrating lymphocytes isolated from lungs with B16-F10 metastases after no treatment, adenovirus (Ad)-DCT + vesicular stomatitis virus (VSV)-DCT, and CTX + Ad-DCT + VSVDCT. (D) Lungs with disseminated melanoma harvested 5 days post boost in mice treated with Ad-DCT + VSV-DCT and CTX + Ad-DCT + VSV-DCT. (E) Kaplan-Meier survival curves of mice with B16-F10 melanoma lung tumors following treatment with the different viral vaccines with or without CTX. Data displayed in (B) and (C) show mean $\pm S D$ of one representative experiment. Data displayed in (E) consist of a pool of five distinct experiments. NS, not significant. 
The B16F10 model is remarkably aggressive, leading to endpoint of untreated animals within 20 days. At the time of CTX preconditioning (day 4), around 10 macroscopic foci of melanoma metastases can be observed in the lungs under a dissecting microscope ${ }^{16}$ Its relevance with regards to the clinic are numerous. ${ }^{17}$ Indeed, $18 \%$ of patients with advanced melanoma develop lung metastases. In particular, approximately $60 \%$ of patients with pulmonary metastatic melanoma are diagnosed with $>3$ nodules; a criterion associated with ineligibility to surgical resection in $70 \%$ of the cases and with poor prognosis. ${ }^{18-20}$ This report builds on previous studies revealing Treg depletion and extended survival times when CTX is used in combination with vaccines in murine models. ${ }^{21}{ }^{22}$ Expanding on these findings, we showed that CTX preconditioning enhances specific $\mathrm{CD}^{+} \mathrm{T}$-cell responses against the TAA targeted by the vaccine and such effects are only observed in the setting of tumor-bearing animals. Intriguingly, we revealed that within secondary lymphoid organs, preconditioning selectively enhances $\mathrm{CD} 4^{+}$and $\mathrm{CD}^{+}$immunity against a TAA, but not against a tumor-irrelevant xenoantigen when tumor-bearing mice were co-vaccinated. Collectively, these results indicate that CTX modulates antitumor immunity by interfering with tumor-induced immunosuppression that relies on interactions between the host's immune system and the immunoevasive neoplastic process. As such, preconditioning with CTX enhances immunity in an antigen-specific manner and future studies are required to determine whether the increases in quantity and quality of immune responses in the tumor-bearing animal can be solely ascribed to Treg depletion.

Regulatory T-cells have been shown to suppress antitumor $\mathrm{CD}^{+}$T-cells in vivo ${ }^{23}$ and an increased ratio of circulating $\mathrm{CD}^{+}$T-cells/Tregs is associated with clinical response to PD1 blockade in patients with advanced melanoma. ${ }^{24}$ Evidence for the positive effect of tumor-infiltrating lymphocyte (TIL) density on melanoma survival times is well established and therapeutic approaches to increase TIL density are therefore soughtafter. ${ }^{25}$ The induction of poly-functional CD8 ${ }^{+} \mathrm{T}$-cells is also beneficial, as such cells were enriched in long-term melanoma survivors following DC vaccination. ${ }^{26}$ In this report, CTX preconditioning favorably improved the ratio of $\mathrm{CD}^{+}$T-cells/Tregs and increased the magnitude of specific multifunctional T-cells within tumor-bearing lungs.

Low-dose CTX before therapeutic cancer vaccination appears well tolerated and has increased survival times in renal cell and biliary tract cancer. ${ }^{2728}$ Based on this report and CTX's favorable therapeutic profile, future clinical evaluation of single, low-dose CTX pretreatment should be considered for patients with melanoma in combination with the next generation of cancer therapeutics, including oncolytic vaccines. ${ }^{5-8} 2930$

\section{Author affiliations}

${ }^{1}$ McMaster Immunology Research Center, Department of Pathology and Molecular Medicine, McMaster University, Hamilton, Ontario, Canada

${ }^{2}$ Equipe 11 labellisée Ligue Nationale contre le Cancer, Centre de Recherche des Cordeliers, Paris, France

${ }^{3}$ Institut National de la Santé et de la Recherche Médicale (INSERM), U1138, Paris, France

${ }^{4}$ Gustave Roussy Cancer Campus, Villejuif, France

${ }^{5}$ Metabolomics and Cell Biology Platforms, Gustave Roussy Cancer Campus, Villejuif, France

${ }^{6}$ Sorbonne Université, Paris, France

${ }^{7}$ Université de Paris, Paris, France

${ }^{8}$ Department of Pathobiology, Ontario Veterinary College, University of Guelph, Guelph, Ontario, Canada

${ }^{9}$ Suzhou Institute for Systems Medicine, Chinese Academy of Medical Sciences, Suzhou, China

${ }^{10}$ Pôle de Biologie, Hôpital Européen Georges Pompidou, AP-HP, Paris, France

${ }^{11}$ Karolinska Institutet, Department of Women's and Children's Health, Karolinska University Hospital, Stockholm, Sweden

Acknowledgements The authors want to thank Liang Zhang for her technical assistance with in vivo experimentations.

Contributors JGP, MJA, KBS, BWB, STW, NK, and AJRM performed the experiments. JGP and MJA wrote the manuscript. BDL, GK, and YW supervised the study and edited the manuscript.

Funding JGP was supported by the Seerave Foundation and the SIRIC Cancer Research and Personalized Medicine (CARPEM). BDL was supported by the Terry Fox Foundation, the Ontario Institute for Cancer Research, BioCanRx and Turnstone Biologics.

Competing interests JGP, MJA, BWB, YW and BDL are named as inventors on patents for cancer vaccination involving an oncolytic rhabdovirus. These patents have been licensed to Turnstone Biologics of which JGP, BWB, YW and BDL are shareholders. KBS is an employee of Turnstone Biologics.

\section{Patient consent for publication Not required.}

Ethics approval All animal studies complied with Canadian Council on Animal Care guidelines and were approved by McMaster University's Animal Research Ethics Board.

Provenance and peer review Not commissioned; externally peer reviewed.

Data availability statement Data are available upon reasonable request. All data relevant to the study are included in the article or uploaded as supplementary information. Raw data remain available upon reasonable request.

Open access This is an open access article distributed in accordance with the Creative Commons Attribution Non Commercial (CC BY-NC 4.0) license, which permits others to distribute, remix, adapt, build upon this work non-commercially, and license their derivative works on different terms, provided the original work is properly cited, appropriate credit is given, any changes made indicated, and the use is non-commercial. See http://creativecommons.org/licenses/by-nc/4.0/.

\section{ORCID iD}

Jonathan G Pol http://orcid.org/0000-0002-8355-7562

\section{REFERENCES}

1 Guo C, Manjili MH, Subjeck JR, et al. Therapeutic cancer vaccines: past, present, and future. Adv Cancer Res 2013;119:421-75.

2 van der Burg SH, Arens R, Ossendorp F, et al. Vaccines for established cancer: overcoming the challenges posed by immune evasion. Nat Rev Cancer 2016;16:219-33.

3 Melief CJM, van Hall T, Arens R, et al. Therapeutic cancer vaccines. J Clin Invest 2015;125:3401-12.

4 Branca MA. Rekindling cancer vaccines. Nat Biotechnol 2016;34:1019-24.

5 Atherton MJ, Stephenson KB, Pol J, et al. Customized viral immunotherapy for HPV-associated cancer. Cancer Immunol Res 2017;5:847-59.

6 Bridle BW, Stephenson KB, Boudreau JE, et al. Potentiating cancer immunotherapy using an oncolytic virus. Mol Ther 2010;18:1430-9.

7 Pol JG, Zhang L, Bridle BW, et al. Maraba virus as a potent oncolytic vaccine vector. Mol Ther 2014;22:420-9. 
8 Bridle BW, Nguyen A, Salem O, et al. Privileged antigen presentation in splenic B cell follicles maximizes $T$ cell responses in prime-boost vaccination. J Immunol 2016;196:4587-95.

9 Krisl JC, Doan VP, Chemotherapy DVP. Chemotherapy and transplantation: the role of immunosuppression in malignancy and a review of antineoplastic agents in solid organ transplant recipients. American Journal of Transplantation 2017:17:1974-91.

10 Abu Eid R, Razavi GSE, Mkrtichyan M, et al. Old-School chemotherapy in immunotherapeutic combination in cancer, a lowcost drug repurposed. Cancer Immunol Res 2016;4:377-82.

11 Le DT, Jaffee EM. Regulatory T-cell modulation using cyclophosphamide in vaccine approaches: a current perspective. Cancer Res 2012;72:3439-44.

12 Sistigu A, Viaud S, Chaput N, et al. Immunomodulatory effects of cyclophosphamide and implementations for vaccine design. Semin Immunopathol 2011;33:369-83.

13 McNally A, Hill GR, Sparwasser T, et al. Cd4+Cd25+ regulatory T cells control CD8+ T-cell effector differentiation by modulating IL-2 homeostasis. Proc Natl Acad Sci U S A 2011;108:7529-34.

14 Emadi A, Jones RJ, Brodsky RA. Cyclophosphamide and cancer: golden anniversary. Nat Rev Clin Oncol 2009;6:638-47.

15 Zhao J, Cao Y, Lei Z, et al. Selective depletion of CD4+CD25+FoxP3+ regulatory T cells by low-dose cyclophosphamide is explained by reduced intracellular ATP levels. Cancer Res 2010;70:4850-8.

16 Sorensen MR, Pedersen SR, Lindkvist A, et al. Quantification of B16 melanoma cells in lungs using triplex Q-PCR--a new approach to evaluate melanoma cell metastasis and tumor control. PLoS One 2014;9:e87831.

17 Overwijk WW, Restifo NP. B16 as a mouse model for human melanoma. Curr Protoc Immunol 2001; Chapter 20:Unit 20.1.

18 Soliman M, Petrella T, Tyrrell P, et al. The clinical significance of indeterminate pulmonary nodules in melanoma patients at baseline and during follow-up chest CT. Eur J Radiol Open 2019;6:85-90.

19 Petersen RP, Hanish SI, Haney JC, et al. Improved survival with pulmonary metastasectomy: an analysis of 1720 patients with pulmonary metastatic melanoma. J Thorac Cardiovasc Surg 2007;133:104-10.
20 Harpole DH, Johnson CM, Wolfe WG, et al. Analysis of 945 cases of pulmonary metastatic melanoma. J Thorac Cardiovasc Surg 1992;103:743-50. discussion 748-750.

21 Liu J-Y, Wu Y, Zhang X-S, et al. Single administration of low dose cyclophosphamide augments the antitumor effect of dendritic cell vaccine. Cancer Immunol Immunother 2007;56:1597-604.

22 Peng S, Lyford-Pike S, Akpeng B, et al. Low-Dose cyclophosphamide administered as daily or single dose enhances the antitumor effects of a therapeutic HPV vaccine. Cancer Immunol Immunother 2013:62:171-82.

23 Chen M-L, Pittet MJ, Gorelik L, et al. Regulatory T cells suppress tumor-specific CD8 T cell cytotoxicity through TGF-beta signals in vivo. Proc Natl Acad Sci U S A 2005;102:419-24.

24 Jacquelot N, Roberti MP, Enot DP, et al. Predictors of responses to immune checkpoint blockade in advanced melanoma. Nat Commun 2017;8:592. 19;8(1):592

25 Lee N, Zakka LR, Mihm MC, et al. Tumour-infiltrating lymphocytes in melanoma prognosis and cancer immunotherapy. Pathology 2016;48:177-87.

26 Wimmers F, Aarntzen EHJG, Duiveman-deBoer T, et al. Longlasting multifunctional $\mathrm{CD}^{+} \mathrm{T}$ cell responses in end-stage melanoma patients can be induced by dendritic cell vaccination. Oncoimmunology 2015;5:e1067745.

27 Shirahama T, Muroya D, Matsueda S, et al. A randomized phase II trial of personalized peptide vaccine with low dose cyclophosphamide in biliary tract cancer. Cancer Sci 2017;108:838-45.

28 Walter S, Weinschenk T, Stenzl A, et al. Multipeptide immune response to cancer vaccine IMA901 after single-dose cyclophosphamide associates with longer patient survival. Nat Med 2012;18:1254-61.

29 Atherton MJ, Stephenson KB, Tzelepis F, et al. Transforming the prostatic tumor microenvironment with oncolytic virotherapy. Oncoimmunology 2018;7:e1445459.

30 Pol JG, Acuna SA, Yadollahi B, et al. Preclinical evaluation of a MAGE-A3 vaccination utilizing the oncolytic Maraba virus currently in first-in-human trials. Oncoimmunology 2019;8:e1512329. 